Discussion: Winter-like photoperiod births in mice induced psychiatryrelevant amotivation as measured by breakpoint, though did not affect social interaction or recognition. In contrast, subchronic PCP treatment increased breakpoint in rats tested 1 and 7 days after treatment. Hence, neurodevelopmental mechanisms underlying the winter-like gestation likely contribute to experiential- but not expressive-related behaviors, while altered NMDA receptor function are unlikely to contribute to such experiential-related abnormalities. Ongoing work will characterize epigenetic, synaptic, and/or system-level adaptations underlying developmental differences between NA and SA photoperiod born mice as well as defining critical periods throughout gestation and rearing that are driving these effects.

\section{F39. MATERNAL IMMUNE ACTIVATION MODELS: MIND YOUR CAGING SYSTEMS!}

\author{
Flavia Müller*,1, Ulrike Weber-Stadlbauer ${ }^{1}$, Urs Meyer ${ }^{1}$ \\ ${ }^{1}$ Institute of Pharmacology and Toxicology, University of Zurich
}

Background: Rodent models of maternal immune activation (MIA) are increasingly used as experimental tools to study neuronal and behavioral dysfunctions in relation to infection-mediated neurodevelopmental disorders such as schizophrenia and autism. One of the most widely used MIA models is based on gestational administration of poly(I:C) (= polyriboinosinic-polyribocytdilic acid), a synthetic analog of double-stranded RNA that induces a cytokine-associated viral-like acute phase response. The effects of poly(I:C)-induced MIA on phenotypic changes in the offspring are known to be influenced by various factors, including the precise prenatal timing, genetic background, and immune stimulus intensity. Thus far, however, it has been ignored whether differences in laboratory housing systems can similarly affect the outcomes of MIA models. Here, we examined this possibility by comparing poly(I:C)-based MIA in two housing systems that are commonly used in preclinical rodent research, namely the individually ventilated cage (IVC) and open cage (OC) systems.

Methods: Pregnant C57BL6/N mice were kept in IVC or OC and treated with a low $(1 \mathrm{mg} / \mathrm{kg}$, i.v.) or high $(5 \mathrm{mg} / \mathrm{kg}$, i.v. $)$ dose of poly(I:C), or with corresponding vehicle solution (pyrogen-free, sterile $0.9 \% \mathrm{NaCl}$; i.v.). MIA or control treatment was induced on gestation day (GD) 9 or 12, and the resulting offspring were raised and maintained in IVC or OC until adulthood for behavioral testing. An additional cohort of dams were used to assess the influence of the different caging systems on poly(I:C)-induced cytokine responses in the maternal plasma, placenta, and fetal brains $1 \mathrm{hr}$ and $6 \mathrm{hrs}$ post-treatment.

Results: Maternal administration of poly(I:C) on GD9 caused a dose-dependent increase in spontaneous abortion in IVC but not in OC system, whereas MIA in IVC systems during a later gestational time-point (GD12) did not do so. Maternal and fetal pro-inflammatory cytokine responses to poly(I:C) were markedly higher in animals kept in IVC as compared to OC systems. The efficacy of MIA to induce long-term behavioral deficits was influenced by the different housing conditions, the dosing of poly(I:C), and the precise prenatal timing.

Discussion: The present study identified the type of cage system as a novel factor that can confound the outcomes of MIA. Our findings thus urge the need to consider and report the kind of cages used in rodent MIA models. Providing this information seems pivotal to yield robust and reproducible results in these models.

\section{F40. NEUREXIN-1A (NRNX1A) HYPOFUNCTION INDUCES SCHIZOPHRENIA-RELEVANT DEFICITS IN CEREBRAL METABOLISM, COGNITIVE PROCESSING SPEED AND COGNITIVE FLEXIBILITY}

Rebecca Hughes*,1, Jayde Whittingham-Dowd ${ }^{1}$, Greg Bristow ${ }^{1}$, Stephen Clapcote ${ }^{2}$, Susan Broughton ${ }^{1}$, Neil Dawson ${ }^{1}$
${ }^{1}$ Lancaster University; ${ }^{2}$ University of Leeds

Background: Heterozygous deletions in NEUREXIN-1 (NRXN1) substantially increase the risk of developing schizophrenia (SZ) (Rujescu et al., 2009. Hum Mol Genet 18(5):988-96). We currently have little understanding into the mechanisms by which NRXN1 impacts on the brain to increase the risk of developing the disorder, and to which symptom domains NRXN1 may contribute. Patients with schizophrenia show deficits in cognitive processing speed and cognitive flexibility (Sanchez et al., 2009. J Clin Psychiat. 70(6):888-896, Dieci et al., 1997. Schizophr Res. 25(1):33-42). In addition, patients show characteristic alterations in brain function including "hypofrontality"; prefrontal cortex hypometabolism (Hill et al., 2004. Acta Psychiatr Scand. 110(4):243-56). Here we characterise, in a transgenic mouse model, the impact of Nrxn1 $\alpha$ hypofunction on cognitive flexibility, processing speed and cerebral metabolism to determine the potential translational relevance of the model to SZ.

Methods: Nrxn1 $\alpha$ heterozygous $(\mathrm{Hz})$ mice and their wild-type (Wt) littermates, of both sexes, were tested at 3, 6,9 and 12 months old using a between-groups design. Associative learning and cognitive flexibility (reversal learning and set shifting) were assessed in a two choice odour based set shifting task (adapted from Young et al., 2010. Cog Affect Behav Neurosci. 10(2):243-251). Mice completed a series of testing phases that included two odour discrimination phases (OD1 and OD2), one reversal learning phase (OD2R) and an extra-dimensional shift (EDS), with animals shifting attentional set from odour to location. The criterion to successfully complete each phase was set at 6 consecutive correct choices. The number of trials to reach criterion, percentage correct and average latency for correct choices were recorded. After behavioural testing cerebral metabolism was determined in 49 brain regions using 14C-2-deoxyglucose functional brain imaging (Dawson et al., 2015. Transl Psychiatry. 5(5):e569). Data were analysed using ANOVA and t-test with Bonferroni correction. Significance was set at $\mathrm{p}<0.05$.

Results: In the associative learning phases of the task (OD1 and OD2) Nrxn $1 \alpha \mathrm{Hz}$ mice took a similar number of trials as Wt controls to reach criteria. Nrxn $1 \alpha \mathrm{Hz}$ mice also completed a similar percentage of correct trials during these phases. This suggests that associative learning is not impaired in $\mathrm{Nrxn} 1 \alpha \mathrm{Hz}$ mice. However, $\mathrm{Nrxn} 1 \alpha \mathrm{Hz}$ mice showed a significant increase in the latency of correct choices in comparison to Wt animals during these phases, supporting significantly decreased processing speed in these animals. We also found that reversal learning (CDR2) was impaired in Nrxn $1 \alpha \mathrm{Hz}$ mice, evidenced by a significant increase in trials to criterion relative to Wt controls. In the brain imaging study, we found that Nrxn1a $\mathrm{Hz}$ mice show significant hypofrontality, with a reduced rate of metabolism in the anterior and medial prelimbic cortex (aPrL, mPrL). By contrast, Nrxn $1 \alpha$ heterozygous mice show significant hypermetabolism in the dorsal raphe (DR), ventral tegmental area (VTA) and retrosplenial cortex (RSC). Discussion: Nrxn $1 \alpha$ heterozygosity induces SZ-like impairments in cognitive processing speed, cognitive flexibility (reversal learning) and cerebral metabolism, including the induction of hypofrontality. Nrxn1 $\alpha$ heterozygosity also alters metabolism in neuromodulatory brain regions, including the serotonergic DR and dopaminergic VTA, which may also contribute to its impact on cognition. These data give new insight into the mechanisms by which NRXN1 heterozygosity increases the risk of developing SZ and suggest that Nrxn $1 \alpha \mathrm{Hz}$ mice provide a translational tool for drug discovery in relation to the cognitive deficits seen in the disorder.

\section{F41. SCHIZOPHRENIA-RELEVANT ALTERATIONS IN CEREBRAL METABOLISM, GLUTAMATE AND MONOAMINERGIC NEUROTRANSMITTER SYSTEM FUNCTION IN A MOUSE MODEL OF 16P11.2 DUPLICATION}

Greg Bristow*,1, David Thomson ${ }^{2}$, Judith Pratt ${ }^{3}$, Brian Morris ${ }^{4}$, Neil Dawson ${ }^{1}$ 